\title{
IMPLEMENTACIÓN DE UN SISTEMA DE GESTIÓN DE LA CALIDAD BASADO EN LA NTC 5581 PARA UN PROGRAMA DE EDUCACIÓN PARA EL TRABAJO Y EL DESARROLLO HUMANO
}

\author{
IMPLEMENTATION OF A QUALITY MANAGEMENT SYSTEM BASED ON THE \\ NTC 5581 FOR A PROGRAM OF EDUCATION FOR WORK AND HUMAN \\ DEVELOPMENT
}

\author{
Yenith Cristina Ortiz González* \\ Christian Camilo Osorio Merchan** \\ Nohra Milena López Sánchez ${ }^{* * * *}$
}

Recibido: 18 de julio de 2017

Aceptado: 14 de noviembre de 2017

\section{Resumen}

Este artículo hace referencia al desarrollo e implementación de programas de formación basados en la norma técnica colombiana NTC 5581 en una institución de educación para el trabajo y el desarrollo humano. Se realiza un diagnóstico del programa de formación de empresa como caso de estudio, con el fin de conocer el grado de cumplimento de los requisitos de la NTC 5581. Posteriormente, se plantea el ciclo PHVA para estructurar la propuesta siguiendo la secuencia lógica, incluyendo los numerales en cada una de las etapas en el ciclo. Dentro de la etapa de planeación se incluyeron: análisis de entorno, definición del perfil de ingreso y egreso de los estudiantes, redacción de competencias y desempeños, estructuración del plan de estudios, métodos de evaluación y selección del personal docente. En la etapa hacer se plantea una metodología de capacitación e implementación, en la que se contempla el compromiso y la participación del personal docente y administrativo. A continuación, en la etapa de verificación se exponen métodos de control y seguimiento. Por último, en la etapa del actuar se establecen unas estrategias para realizar acciones de mejora basadas en el análisis de la etapa de verificación.

Palabras clave: aprendizaje basado en competencias, competencias laborales, programas de formación, NTC 5581, diseño curricular a partir de competencias.

\footnotetext{
* Ingeniera industrial, magíster en Calidad y Gestión Integral, especialista en Gestión de Productividad y Calidad, estudiante de Doctorado en Educación. Docente investigadora, Centro de Investigación en Competitividad Empresarial, Fundación Universidad de América, Bogotá, Colombia. ORCID: http://orcid.org/0000-0003-3601-6517 induscris@yahoo.es/ yenith.ortiz@ investigadores.uamerica.edu.co

** Ingeniero mecánico, especialista en Gerencia de la Calidad. Centro de Investigación en Competitividad Empresarial, Fundación Universidad de América, Bogotá, Colombia. christian_osorio @ outlook.com

*** Ingeniera Industrial, especialista en Planeación Educativa, magíster en Docencia, estudiante de Doctorado en Administración. Docente Investigadora de la Universidad Católica, Bogotá, Colombia. ingnohralopez@gmail.com, nmlopez@ucatolica.edu.co
} 


\section{Abstract}

This article refers to the development and implementation of training programs based on the Colombian technical standard 5581 (NTC in Spanish) in an institution of education for work and human development. A diagnosis of the company training program is made as a case study, to understand the degree of compliance with the requirements of NTC 5581. Subsequently, the PDCA cycle to structure the proposal following the logical sequence, including numerals at each stage in the cycle. The planning stage included: environmental analysis, the definition of students' entry and exit profiles, writing skills and performances, structuring the curriculum, methods of assessment and selection of teaching staff. The doing stage approaches a training methodology and implementation, in which envisages the commitment and involvement of teachers and administrative staff. Then, in the checking stage, control and monitoring methods are exposed. Finally, at the Acting stage sets out strategies for improvement actions based on the analysis of the checking phase.

Keywords: competency-based learning, job skills, training programs, NTC 5581, curriculum design based on competencies.

\section{INTRODUCCIÓN}

La educación, uno de los motores del desarrollo del ser humano y de cualquier nación, permite mejorar la calidad de vida en cuanto implica mejores ingresos, así como impulsa el desarrollo de las áreas del saber, la investigación, la tecnología, la innovación y la economía. Por tal motivo es de gran importancia garantizar la calidad de los programas de formación.

Con el fin de diseñar y desarrollar programas de formación, se debe tener claro los métodos de aprendizaje, debido que los seres humanos somos dinámicos y aprendemos de maneras distintas. Al respeto, López (2013) señala:

La manera en que aprenden las personas es dinámica, lo hacen desde que nacen hasta que mueren, de forma compleja, sistémica, multisensorial, creativa, transdisciplinar y valoral. Desde esta perspectiva, [...] el aprendizaje puede ser visto bajo una nueva dimensión, que parte de un proceso sistémico, que es circular o recursivo y que, a manera de bucle, ha de crear una serie de ciclos y apropiación de ideas, procesos, relaciones e interacciones con la realidad, durante un periodo de que se extiende permanentemente. (p 2)

Para que exista un desarrollo del conocimiento, se debe tener en cuenta dos tipos de conocimientos: de dominio (superficial) y estratégico (profundo). López (2013) establece que el conocimiento de dominio representa "el llamando conocimiento factico y procedimental; se trata del conocimiento visible, explícito o ejecutable, el que se puede poner en práctica ante los ojos de los demás" (p 4). Por su parte, el conocimiento estratégico representa:

[...] habilidades de orden superior, no visibles, que conforman lo que ha denominado conocimiento tácito. En este tipo de conocimiento sobresalen los métodos que los expertos utilizan para resolver problemas al abordar situaciones de la vida cotidiana. Este conocimiento estratégico es señalado como el conocimiento que reside en la acción, vinculado con el saber cómo. Este conocimiento forma parte de las habilidades tacitas del experto al hacer uso de conceptos, hechos y procedimientos, según se necesiten para resolver problemas o llevar a cabo actividades de su especialización. (p. 5) 
Este tipo de conocimiento no es fácil de comunicar, puesto que: "es de acceso difícil para los aprendices y de amplio dominio de los expertos" ( $\mathrm{p} 5$ ).

López (2013) también estable que "el conocimiento de dominio se relaciona con saber demasiado sobre un tema” ( $\mathrm{p}$ 6). Dentro del proceso de construcción de conocimiento estratégico, se propone el llamado modelo de espiral práctica, el cual, según el autor, se desarrolla en tres momentos:

En el primero, el experto se encarga de explicar y demostrar un evento, un suceso, un proceso, un caso; posteriormente el experto apoya para que los participantes reflexionen acerca de los aprendido; se encarga revisar el progreso de los participantes, finalmente lleva acabo la planeación para volver a mostrar la practicas. En el segundo momento de la espiral, el novato (a través de la supervisión del experto) practica lo enseñado, reflexiona sobre su desempeño, revisar el procedimiento requerido y planea sus acciones futuras. En un tercer momento el proceso anterior se repite, cada vez con menor supervisión, hasta que el novato logra alcanzar el progreso deseado al practicar, reflexionar, revisar y planear su desempeño. El experto se hace cargo de valorar el trabajo llevado a cabo por medio del análisis de evidencias desarrolladas por los novatos en cada una de las prácticas. (p. 6)

Con el fin de generar un aprendizaje significativo y desarrollar las competencias requeridas por el sector productivo, se debe disponer de un modelo pedagógico que oriente el proceso formativo del estudiante y estipule una guía para el docente. El enfoque basado en competencias es uno de los modelos para este fin, porque permite desarrollar los aprendizajes:

[...] es considerado como un enfoque educativo que hace evidente el aprendizaje de conocimiento (el qué), el desarrollo de habilidades (el cómo), así como una serie de actitudes y valores en una situación determinada (el para qué), todos ellos factores para un desempeño del acto educativo. (López, 2013, p. 55)

Antes de continuar con los modelos pedagógicos por competencias, es importante definir qué es una competencia y qué tipos existen. Las competencias son, según Santivañez (2013):

[...] capacidades o habilidades (qué) para efectuar tareas o hacer frente a situaciones diversas (para qué) de forma eficaz (de qué manera) en un contexto determinado, (dónde), siendo para ello necesario movilizar actitudes, habilidades y conocimientos (por medio de qué) al mismo tiempo y de forma interrelacionada (cómo). (p. 137)

Las competencias tienen tres componentes, los cuales se relacionan entre sí para movilizar los distintos recursos, como las capacidades y habilidades en un contexto real. En la tabla 1 se muestran estas tres dimensiones.

TABLA 1. Dimensiones de las competencias

- Saber conocer: conocimientos factuales y declarativos

- $\quad$ Saber hacer: habilidades, destrezas y procedimientos

- $\quad$ Saber ser: actitudes y valores

Fuente: basado en Pimienta (2012). 
Igualmente, Pimienta (2012) resalta que:

[...] las competencias existen por la necesidad de resolver problemas y situaciones. Cuando se realiza un diseño de curricular basado en competencias, los problemas de la profesión hacen necesarias las competencias. (p 5)

De acuerdo con la definición y según en el proyecto Tuning, ${ }^{11}$ se distinguen dos tipos de competencias: genéricas y específicas; estas a su vez se dividen en tres tipos: instrumentales, interpersonales y sistemática.

Competencias especificas. El conocimiento concreto de cada área temática (López, 2013, p. 40).

Competencias genéricas. "También conocidas como trasversales, se trata de competencias comunes que involucran conocimientos transferibles” (p. 40). Las competencias genéricas están relacionadas con tres saberes (véase tabla 2).

TABLA 2. Dimensiones de las competencias

- Saber conocer: incluye conocimiento general y específicos de una disciplina. También se asocia al domino de métodos y técnicas.

- Saber ser: involucra las actitudes y formas de actuar e interactuar con otras personas. Tiene que ver con posturas personales relacionadas con la iniciática, la motivación y el liderazgo.

- Saber actuar: se vincula con la formación permanente, la planeación y ejecución creativa de un problema, un caso o un proyecto.

Fuente: López (2013).

Se pretende entonces que el alumno "movilice su saber poniendo en práctica lo aprendido, nos solo dentro de un salón de clases, sino que lo contextualice y lo logre trasferir a diversas situaciones a lo largo de toda su vida" (p.54). También es importante resaltar que:

El aprendizaje basado en competencias es considerado como un enfoque educativo que hace evidente el aprendizaje de conocimiento (el qué), el desarrollo de habilidades (el cómo), así como una seria de actitudes y valores en una situación determinada (el para qué), todos ellos factores para un desempeño del acto educativo. ( $\mathrm{p} 55)$.

Considerando anterior, una competencia puede ser definida "como una saber de ejecución, vinculado un saber pensar, saber desempeñar, saber interpretar, así como saber actuar en escenarios diversos"(López, 2013, p. 57).

Para el diseño y planeación de un programa con enfoque en competencias se deben considerar las metas u objetivos de aprendizaje, que son las capacidades que el estudiante debe desarrollar al terminar el módulo, curso o programa; una vez determinadas las metas, se deben identificar los resultados y las competencias desarrolladas. Para identificar el propósito del aprendizaje deben tenerse en cuenta las preguntas establecidas en la tabla 3.

${ }^{1}$ El proyecto Alfa Tuning América Latina busca "afinar" las estructuras educativas de América Latina iniciando un debate cuya meta es identificar e intercambiar información y mejorar la colaboración entre las instituciones de educación superior para el desarrollo de la calidad, efectividad y transparencia. Es un proyecto independiente, impulsado y coordinado por universidades de países latinoamericanos y europeos (http://tuning.unideusto.org/tuningal/) 
TABLA 3. Identificación de propósitos de aprendizaje

¿Qué es lo que se desea que los estudiantes sean capaces de hacer, saber y comprender?

¿Cuáles son los contenidos con los que se necesita familiarizar a los estudiantes?

¿Qué procedimientos, técnicas o métodos requieren saber usar o utilizar?

¿Qué habilidades de pensamiento de orden superior-nivel de comprensión-quiere que sus estudiantes desarrollen en el curso o programa?
- Propósitos de aprendizaje.

- El qué (el saber saber)

- El cómo (el saber hacer)

- El para qué, el cuándo, el dónde

(el saber comprender, transferir o contextualizar).

Fuente: López (2013).

Como se explicó, el aprendizaje basado en competencias implica el desarrollo de saberes, dentro de lo cual es importante resaltar cómo el estudiante pone en práctica lo aprendido en situaciones de vida personal, social y profesional. Para desarrollar adecuadamente los saberes, la institución tiene que tener en cuenta una jerarquización de niveles o categorías de contenidos y planear las preguntas sobre cómo se estipula en cada uno de los siguientes saberes:

El saber qué. Este saber involucra la presentación de datos y análisis de información, con el fin de que los estudiantes posteriormente la interpreten y evalúen. En la tabla 4 se plantea una serie de preguntas que orientan el desarrollo de este saber.

TABLA 4. Identificación del saber qué

\section{Pregunta}

¿Qué contenidos (hechos, detalles, conceptos, terminología) necesitaran familiarizarse los estudiantes en el curso?

¿Cuáles son los saberes fundamentales?

¿Qué contenidos -temas y subtemas- garantizan el nivel de conocimiento requerido?

¿Qué información se considera como mínima indispensable y que de ninguna manera se tiene que omitir?

¿Qué información se considera secundaria y se puede de alguna forma sustituir o eliminar?

$$
\text { Fuente: López (2013). }
$$

El saber hacer (el cómo). Permite poner en práctica la comprensión de los conocimientos. Es un elemento esencial para el desarrollo de competencias. Al respecto, López (2013) señala: "es fundamental que los estudiantes prueben, manipulen, experimenten, resuelvan, demuestren y lleven a cabo una serie de situaciones prácticas" ( $\mathrm{p}$ 64). En la tabla 5 se plantean preguntas asociadas a este saber.

TABLA 5. Identificación del saber cómo.

\section{Preguntas}

Como resultado de los conocimientos adquiridos: ¿Qué deberían de ser capaces de hacer los estudiantes?

¿Cuáles experiencias de aprendizaje son necesarias para que los estudiantes prueben, manipules, experimenten, resuelvan, demuestren y lleven a cabo una serie de situaciones prácticas?

¿Cuáles recursos y de qué manera deberían ser utilizados para alcanzar la compresión?

¿Cuáles estrategias de aprendizaje y de qué manera deberán ser movilizadas para aprender, actuar y reflexionar?

¿Qué procedimientos, técnicas o métodos deber ser capaces de aplicar los estudiantes?

Fuente: López (2013). 
Para lograr desarrollar el saber cómo, los estudiantes deben pasar por distintas categorías; a su vez, el docente y la institución deben prever los ambientes para lograrlo, es decir, es necesario que en el programa se planifiquen estrategias que permitan comprender y aplicar los conceptos estipulados en el saber qué.

El saber ser (el para qué). Permite darle sentido a los contenidos del saber qué y el saber hacer, ya que, según Santivañez (2013): "son prácticas sociales a través de las cuales se manifiestan los ideales de los estudiantes y las normas de convivencia” (p. 148). Según López (2013), este saber permite que el estudiante "identifique el cuándo y dónde, esto es, en qué condiciones se debe haber esto y no lo otro; ayuda a determinar cuándo hacer las cosas, por qué y bajo qué tipo de circunstancias" (p. 66). En la tabla 6 se plantean una serie de pregunta para definir el saber ser.

TABLA 6. Identificación del saber ser

$\frac{\text { Preguntas }}{\text { ¿De qué manera específica se espera que el estudiante comprenda, transfiera o contextualice? }}$
¿En términos de la comprensión, qué nivel espera que sus estudiantes alcancen?
¿Qué habilidades de pensamiento complejo espera que los estudiantes desarrollen en este curso?

Fuente: López (2013).

Para diseñar un programa a partir de competencias es importante considerar los recursos, las actividades de aprendizaje y los métodos de evaluación.

En el caso del sector educativo, las certificaciones en calidad permiten que las instituciones se visualicen con mayor facilidad, y al igual que ocurre con las empresas, puedan posicionarse en el mercado como un eje de desarrollo nacional (Díaz, 2013).

Es de aclarar que la NTC 5581 sigue una estructura similar a la NTC ISO 9001. Por lo cual según (Ortiz y Ramírez, 2017) El estándar ISO 9001 sigue siendo tendencia en implementación dentro de las organizaciones, como respuesta a las exigencias presentadas para la participación dentro de mercados nacionales e internacionales.

Es por ello que las instituciones de educación superior son impulsadas por el Ministerio de Educación Nacional (2016), quien asegura que las certificaciones abren posibilidades a nivel internacional de los programas y los métodos y procesos de acreditación.

\section{Metodología}

La metodología utilizada se basó en dos aspectos.

\section{Aspecto 1. Diagnóstico}

El diagnóstico es un instrumento que permite evidenciar el estado de los programas con respecto a los requisitos de la NTC 5581; para tal fin se realizó una lista de verificación, en la que se evaluaron cada uno de los numerales y se compararon con respecto a lo que la institución y el Proyecto Educativo Institucional (PEI).

\section{Aspecto 2. Implementación de norma NTC 5581-2011}

A partir de los resultados del diagnóstico se inició la implementación de la NTC 5581 (Icontec, 2011b), teniendo en cuenta el planear, el hacer, el verificar y el actuar (PHVA). 
Según Singh, (1997), el ciclo PHVA es muy similar al ciclo Deming. Los términos planear, hacer, verificar y actuar (PHVA) definen cada una de las etapas: 1) planear: determinar las metas y los métodos para alcanzar esas metas; 2) hacer: educar a los empleados y poner en práctica el cambio; 3) verificar: verificar los efectos del cambio: ¿se han alcanzado las metas? (de no ser así, volver a la etapa de planear); 4) actuar: emprender la acción apropiada para institucionalizar el cambio.

\section{Resultados}

A partir de los resultados del diagnóstico, los numerales que requieren mayor atención son 4.3, 4.5, 4.6, 4.7 y 4.8, estos no están implementados completamente o se encuentran de manera parcial (ver figura 1). Para dar repuesta a los numerales mencionados, se empleó el ciclo PHVA.

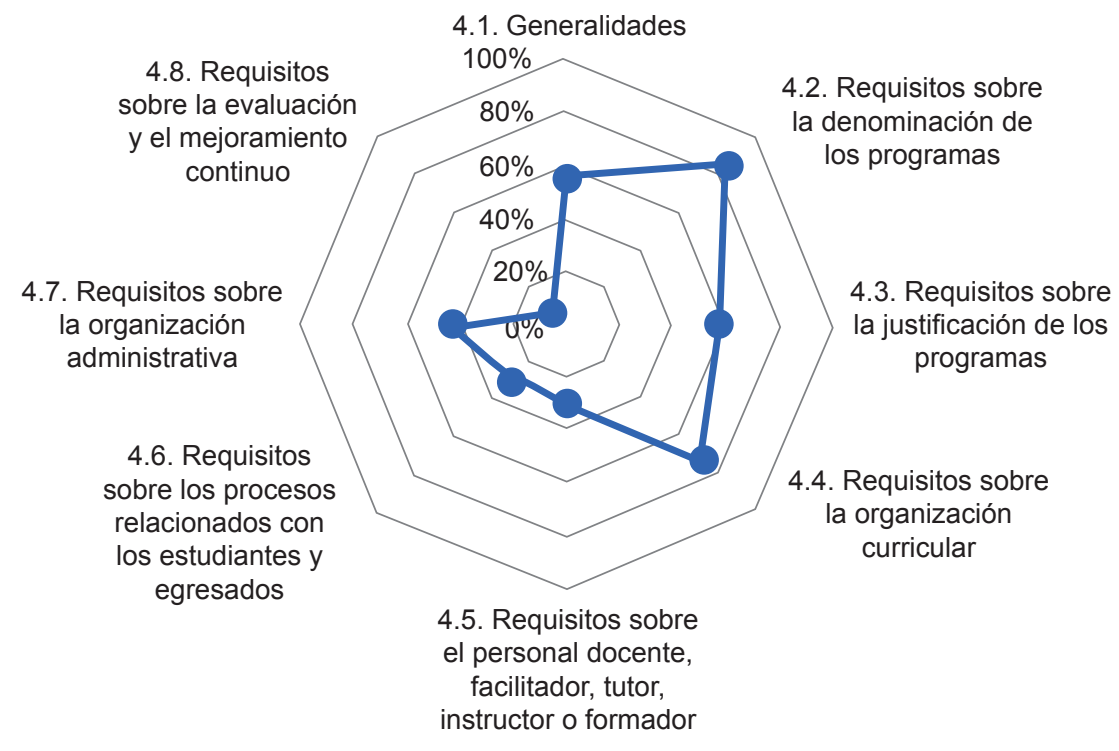

Figura 1. Diagnóstico de la institución.

Es importante resaltar que el PEI, el marco legal y las normas de competencias aportan información para el desarrollo de los programas; igualmente, en algunos numerales con solo cumplir los requisitos legales establecidos se logra la apertura de una institución educativa y simultáneamente se están cumpliendo con algunos requisitos de la norma.

Teniendo en cuenta los resultados del diagnóstico, se abordó la implementación de la norma paso a paso con base en el ciclo del PHVA.

\section{Etapa de planeación}

Según la NTC 5555 (Icontec, 2011a), la planeación es la etapa donde se debe:

[...] definir lo que se espera que alcancen los estudiantes, en relación con los requisitos del sector productivo. Para el caso de la formación para el trabajo, se requiere establecer las competencias que obtendrán los estudiantes en relación con la Clasificación Nacional de Ocupaciones-CNO y que estén definidas en normas de competencia laboral por las mesas sectoriales". (p 56)

En esta etapa se define el qué y el cómo, es decir, cuál es el objetivo y cómo lograrlo. 
TABLA 7. Pasos en la etapa de planeación

\begin{tabular}{|c|c|}
\hline N. ${ }^{\circ}$ del paso & Elementos a tener en cuenta \\
\hline Justificación del programa & $\begin{array}{l}\text { - Marco legal } \\
\text { - Estudios de caracterización } \\
\text { - Mapas funcionales } \\
\text { - }- \text { Normas de competencia } \\
\text { - Consultas con el sector productivo } \\
\text { - Análisis de tendencias ocupaciones } \\
\text { - Necesidades de formación } \\
\text { - PEl } \\
\text { - Misión y visión de la institución }\end{array}$ \\
\hline Definir los perfiles de ingreso y egreso & $\begin{array}{l}\text { - Marco legal } \\
\text { - Competencias básicas } \\
\text { - Competencias ciudadanas } \\
\text { - Normas de competencias } \\
\text { - Competencias laborales generales y específicas } \\
\text { - Clasificación nacional de ocupaciones } \\
\text { - PEI } \\
\text { - Anexo A }\end{array}$ \\
\hline Enfoque pedagógico y metodológico & $\begin{array}{l}\text { - PEI } \\
\text { - Enfoque en competencias } \\
\text { - Marco legal }\end{array}$ \\
\hline Plan de estudios & $\begin{array}{l}\text { - Organización de las actividades } \\
\text { - Estrategia metodología } \\
\text { - Distribución del tiempo } \\
\text { - Evaluación de los aprendizajes } \\
\text { - Recursos del programa } \\
\text { - Competencias del personal docente } \\
\text { - Asignación académica } \\
\text { - Vinculación sector productivo }\end{array}$ \\
\hline
\end{tabular}

\section{Paso 1. Justificación del programa}

La justificación permite conocer la realidad del sector productivo, las tendencias de la educación, las demandas de los profesionales en determinado sector, los campos de aplicación, las áreas ocupaciones y las oportunidades de empleabilidad, y debe ser coherente con la misión-visión y el PEI. Por otro lado, según NTC 5555 (Icontec, 2011), la justificación permite establecer los "criterios para ofrecer un programa de formación: demandas del sector productivo, pertinencia en relación con las necesidades del contexto y oportunidades de desempeño de los egresados" (p. 5). 
TABLA 8. Elementos de la justificación de un programa

\begin{tabular}{|c|c|c|}
\hline Elemento & Descripción & Actividades a realizar \\
\hline $\begin{array}{l}\text { Estudio de la } \\
\text { realidad }\end{array}$ & $\begin{array}{l}\text { Para realizar esta etapa se debe } \\
\text { investigar y recopilar información del } \\
\text { entorno social, educativo y profesional } \\
\text { de sector en el que se identificó una } \\
\text { necesidad de formación. }\end{array}$ & $\begin{array}{l}\text { - Realizar entrevistas y encuestas en el sector productivo. } \\
\text { - Identificar las tendencias nacionales e internacionales de } \\
\text { profesión. } \\
\text { - Demandas económicas y sociales de la profesión. } \\
\text { - Identificar el objetivo de la profesión y el campo de } \\
\text { ocupación. }\end{array}$ \\
\hline $\begin{array}{l}\text { Marco } \\
\text { normativo }\end{array}$ & $\begin{array}{l}\text { Por un lado, las normas de } \\
\text { competencias laborales son un } \\
\text { referente para el diseño de programas } \\
\text { y, por otro, el marco legal permite } \\
\text { cumplir con los requisitos legales. }\end{array}$ & $\begin{array}{l}\text { - Revisar las normas de competencias laborales. } \\
\text { - Verificar la clasificación nacional y la internacional de } \\
\text { ocupaciones. } \\
\text { - Considerar el aspecto legal en cuanto a los } \\
\text { requerimientos del programa como al ejercicio de la } \\
\text { profesión. }\end{array}$ \\
\hline Análisis DOFA & $\begin{array}{l}\text { El análisis de DOFA permite: } \\
\text { identificar a los competidores; valorar } \\
\text { las fortalezas y debilidades de la } \\
\text { institución, y analizar las amenazas y } \\
\text { las oportunidades externas que puede } \\
\text { influir el programa y el ejercicio de la } \\
\text { profesión }\end{array}$ & $\begin{array}{l}\text { - Investigar las competidores que ofrecen el mismo o un } \\
\text { programa similar de formación. } \\
\text { - Reunirse con el área administrativa y docente, con el fin } \\
\text { de evaluar los factores internos de la organización } \\
\text { - Construir la matriz DOFA para de evaluar la viabilidad del } \\
\text { programa. }\end{array}$ \\
\hline
\end{tabular}

\section{Paso 2. Definir los perfiles de ingreso y egreso}

Los perfiles de ingreso permiten estipular los requisitos mínimos para ingreso y el desarrollo normal del proceso formativo. Según el Decreto 1075 de 2015, "son los requisitos para el ingreso a los diferentes programas de educación para el trabajo y el desarrollo humano los que señale cada institución de acuerdo con el programa que va a desarrollar y el perfil ocupacional de egreso" (art. 2.6.4.4). Es importante resaltar que el perfil de ingreso se construye una vez se defina el perfil de egreso, ya que lo que se debe considerar son los desempeños del egresado para el posterior desarrollo del programa formativo.

Por otro lado, el perfil de egreso permite establecer las competencias, conocimientos y habilidades que la institución se compromete a desarrollar y que son necesarios para ejercer la profesión; igualmente, estos deben estar en concordancia con las necesidades de formación del sector productivo. El perfil profesional del egresado, según Santivañez (2013):

[...] es el conjunto de las competencias genéricas y específicas que la institución educativa va formar en los estudiantes, a partir de la revisión de la profesión y del estudio de actividades y problemas hallados en el análisis del contexto disciplinar, investigativo, social y profesional-laboral. (p. 70) 
TABLA 9. Agrupación de competencias

\begin{tabular}{|c|c|c|}
\hline Grupo & Características & Tipo de competencia \\
\hline $\begin{array}{l}\text { Competencias } \\
\text { genéricas }\end{array}$ & $\begin{array}{l}\text { Según Santivañes (2013): "estas se entienden como } \\
\text { las competencias transversales, es decir que engloban } \\
\text { capacidades referidas a la interacción humana, } \\
\text { como, por ejemplo: la capacidad de aprender, tomar } \\
\text { decisiones, planificar, etc." (p. 74). }\end{array}$ & $\begin{array}{l}\text { Competencias ciudadanas } \\
\text { Competencias ambientales } \\
\text { Competencias laborales genéricas } \\
\text { Competencias de fundamentación } \\
\text { tecnológica } \\
\text { Competencias proyecto Tuning }\end{array}$ \\
\hline $\begin{array}{l}\text { Competencias } \\
\text { específicas }\end{array}$ & $\begin{array}{l}\text { Según NTC } 5581 \text { (Icontec, 2011): "competencia orientada } \\
\text { a habilitar a las personas para desarrollar funciones } \\
\text { productivas propias de una ocupación o funciones } \\
\text { comunes a un conjunto de ocupaciones"(p. 3). }\end{array}$ & Competencias laborales especificas \\
\hline
\end{tabular}

Para la definición de una competencia específica se debe tener en cuenta el campo ocupacional, el cargo y el domino del desempeño; una vez considerado lo anterior, se debe escribir tal como se puede apreciar en el paso 5 de la tabla 11. La matriz formulación del perfil del egresado a partir de competencias específicas (ver tabla 10) permite vincular los elementos mencionados y adicionar un componte importante en la definición del perfil.

TABLA 10. Matriz formulación del perfil del egresado a partir de competencias especificas

\begin{tabular}{lllll}
\hline \multicolumn{1}{c}{$\begin{array}{c}\text { Campos } \\
\text { ocupacionales }\end{array}$} & $\begin{array}{c}\text { Puestos } \\
\text { de trabajo } \\
\text { (cargo) }\end{array}$ & $\begin{array}{c}\text { Dominios de } \\
\text { desempeño }\end{array}$ & $\begin{array}{c}\text { Competencias } \\
\text { especificas }\end{array}$ & Desempeños específicos \\
\hline $\begin{array}{l}\text { Son los lugares } \\
\text { donde se va } \\
\text { desempeñar el } \\
\text { egresado }\end{array}$ & $\begin{array}{l}\text { Son las } \\
\text { responsabilidades } \\
\text { que asumirá el }\end{array}$ & $\begin{array}{l}\text { VERBO }+ \\
\text { OBJETO + } \\
\text { CONDICIÓN }\end{array}$ & $\begin{array}{l}\text { Acciones que el estudiante debe } \\
\text { realizar para demostrar que ha lograda } \\
\text { dominar y desarrollar con eficacia } \\
\text { y eficiencia la primera competencia } \\
\text { especifica. }\end{array}$ \\
\hline
\end{tabular}

TABLA 11. Etapas formulación del perfil de egreso

\begin{tabular}{|c|c|c|}
\hline Paso & & Descripción \\
\hline \multirow{14}{*}{$\begin{array}{l}\text { 1. Diagnóstico de la } \\
\text { demanda }\end{array}$} & \multirow{5}{*}{$\begin{array}{l}\text { a. Caracterización de la sociedad y } \\
\text { su perspectiva de desarrollo }\end{array}$} & Situación actual de la sociedad \\
\hline & & Prognosis económica del último quinquenio \\
\hline & & La imagen objetiva \\
\hline & & La estrategia nacional de desarrollo \\
\hline & & Matricula en el nivel por especialidades \\
\hline & \multirow{3}{*}{$\begin{array}{l}\text { b. Oferta actual de recursos } \\
\text { calificados }\end{array}$} & Matriculas en el nivel por especialidades y por regiones \\
\hline & & Población económicamente activa por sectores \\
\hline & & Sectores económicos prioritarios en la oferta \\
\hline & \multirow{2}{*}{$\begin{array}{l}\text { c. Áreas prioritarias en la demanda } \\
\text { de recursos humanos calificados }\end{array}$} & Comportamiento de la PEA \\
\hline & & $\begin{array}{l}\text { Áreas o sectores prioritarios en la demanda: plan a } \\
\text { mediano plazo, plan a largo plazo }\end{array}$ \\
\hline & \multirow{4}{*}{ d. Demanda de recursos humanos } & Empleo regional por sectores económicos \\
\hline & & Empleo regional por niveles y categorías ocupacionales \\
\hline & & Planes de desarrollo y demanda potencial de RR. HH \\
\hline & & $\begin{array}{l}\text { Prioridad de formación profesional por campos o grupos } \\
\text { ocupacionales }\end{array}$ \\
\hline
\end{tabular}




\begin{tabular}{|c|c|c|}
\hline Paso & & Descripción \\
\hline \multirow{2}{*}{$\begin{array}{l}\text { 2. Determinación de } \\
\text { los componentes del } \\
\text { perfil profesional del } \\
\text { egresado }\end{array}$} & $\begin{array}{l}\text { Análisis y diagnóstico de la nueva } \\
\text { actividad de la profesión (nivel } \\
\text { macro y micro) }\end{array}$ & $\begin{array}{l}\text { Análisis de profesionales exitosos en actividades de la } \\
\text { carrera } \\
\text { Análisis de profesionales sin éxito o subempleados } \\
\text { Análisis de cliente de ambos profesionales }\end{array}$ \\
\hline & $\begin{array}{l}\text { Análisis y diagnóstico de las } \\
\text { instituciones que forman a los } \\
\text { profesionales cuyo perfil se está } \\
\text { estructurando }\end{array}$ & $\begin{array}{l}\text { Análisis de la organización y el contenido curricular } \\
\text { Análisis del personal docente } \\
\text { Análisis de la infraestructura y tecnología disponible } \\
\text { para la formación }\end{array}$ \\
\hline \multirow{2}{*}{$\begin{array}{l}\text { 3. Determinación de } \\
\text { la metodología de } \\
\text { análisis ocupacional }\end{array}$} & Análisis ocupacional Dacum & $\begin{array}{l}\text { Análisis del puesto de trabajo y la tarea para definir el } \\
\text { currículo de formación. Descripción de tarea }\end{array}$ \\
\hline & $\begin{array}{l}\text { Elaboración matriz de análisis } \\
\text { ocupacional }\end{array}$ & $\begin{array}{l}\text { Elaboración de una matriz donde se especifiquen los } \\
\text { campos ocupacionales, puesto de trabajo y dominios de } \\
\text { desempeño }\end{array}$ \\
\hline
\end{tabular}

Se toma en cuenta el VERBO u OPERACIÓN, lo que implica señalar la cualidad de la acción, para cuyo fin se utiliza la taxonomía de objetivos

Se indica el OBJETO, es decir, se precisan los contenidos curriculares conformando por el aspecto cognitivo, procedimental y/o actitudinal

4. Formulación del perfil genérico del profesional egresado

Se complementa con la CONDICIÓN o SITUACIÓN DE CONTEXTO, que se refiere a la aplicación personal, social, académica y/o laboral
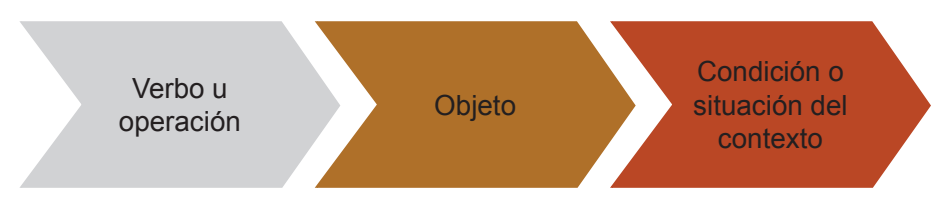

Se toma en cuenta el VERBO u OPERACIÓN, el cual implica señalar la cualidad de la acción, para cuyo fin se utiliza la taxonomía de objetivos

Redacción competencias específicas

5 Formulación del perfil específico del egresado
Elaboración de matrices de competencias y desempeños

Formulación de los desempeños específicos
Se indica el OBJETO, es decir, se precisan los contenidos curriculares conformando por el aspecto cognitivo, procedimental y/o actitudinal

Se complementa con la CONDICIÓN o SITUACIÓN DE CONTEXTO, que se refiere a la aplicación personal, social, académica y/o laboral

Se elaboran a partir de la matriz de análisis ocupacional (paso 3), y se tiene cuenta los dominios de desempeño laboral, competencias específicas y desempeños específicos

Identificar las acciones que debe realizar el educando para demostrar que ha logrado dominar y desarrollar con eficacia y eficiencia las competencias

\section{Paso 3. Enfoque pedagógico y metodológico}

Con el fin de desarrollar un aprendizaje significativo y lograr las competencias estipuladas en el perfil de egreso, la institución debe definir un enfoque pedagógico y metodológico. El enfoque propuesto es el aprendizaje basado en competencias, el cual permite centrar el protagonismo en el estudiante y enfrentarlo a situaciones reales con el fin de poner en práctica lo aprendido. 
TABLA 12. Aspectos enfoque pedagógico y metodológico

\begin{tabular}{lll}
\hline \multicolumn{1}{c}{ Aspectos } & Dominios & Saberes \\
\hline El qué (pensar) & Conocimientos & Saber conocer \\
El cómo (actuar) & Desarrollo de habilidades & Saber hacer \\
El para qué o dónde & Comprensión & Saber convivir y ser \\
\hline
\end{tabular}

Para desarrollar competencias de deben tener en cuenta tres etapas:

1. Determinar los objetivos que aseguren que los estudiantes conozcan, hagan y transfieran los conocimientos estipulado del programa en la realidad actual.

2. Establecer los medios, evidencias y recursos que permitan alcanzar las metas propuestas, para lo que es necesario la planeación y desarrollo de estrategias y actividades de aprendizaje.

3. Disponer de un método de evaluación que permita la valoración del desempeño del estudiante en cada una de las asignaturas.

Un elemento importante para lograr los anteriores saberes es la guía de aprendizaje. Según López (2013), esta guía:

[es] un instrumento que orienta el proceso de aprendizaje basado en competencias, el cual se aborda mediante la determinación de metas, el establecimiento del medio necesario para la promoción del aprendizaje y una propuesta de evaluación, en la que se identifiquen evidencias de aprendizaje. (p.79)

El desarrollo de la guía de aprendizaje se profundizará en el paso 4.

\section{Paso 4. Planes de estudios}

En este paso se propone una metodología para diseñar las experiencias de aprendizaje, que son formuladas según el perfil de egreso y las competencias que el estudiante requiere desarrollar. Dentro del plan de estudios se encuentran: el conjunto de actividades de formación, las estrategias metodológicas, la distribución del tiempo, la evaluación de los aprendizajes, los contextos y los recursos de aprendizaje. Para el desarrollo de la metodología se tiene en cuenta las etapas establecidas en la tabla 13.

TABLA 13. Aspectos enfoque pedagógico y metodológico

\begin{tabular}{ll}
\hline 1. & Cuadro de asignaturas \\
2. Mapa curricular \\
3. Datos de la asignatura \\
4. Sumillas \\
5. Objetivo de la asignatura \\
6. Competencias de la asignatura \\
7. Organización de la asignatura \\
8. Plan de aprendizaje \\
9. Normas de convivencia
\end{tabular}


Cuadro de asignaturas. Permite organización de manera secuenciada las asignaturas que debe estudiar el futuro profesional cumpliendo con el perfil de egreso. Para elaborar un cuadro de asignaturas, Santivañez (2013) propone elaborar una matriz de identificación de las asignaturas a partir del perfil de egreso (véase tabla 14). Una vez identificados todos los temas, se agrupan y se nombra la asignatura. Conocidas las asignaturas, se crea un cuadro donde se distribuyen de acuerdo a: áreas curriculares, ciclo académico y la condición (obligatoria o electiva). En la tabla 15 se propone un esquema para la distribución de asignaturas.

TABLA 14. Matriz de identificación de las asignaturas a partir del perfil específico del egresado

\begin{tabular}{|c|c|c|c|c|c|c|}
\hline $\begin{array}{l}\text { Dominios de } \\
\text { desempeño }\end{array}$ & $\begin{array}{l}\text { Competencias } \\
\text { especificas }\end{array}$ & $\begin{array}{l}\text { Desempeños } \\
\text { específicos }\end{array}$ & Contenidos & Asignaturas & $\begin{array}{c}\text { Dimensiones } \\
\text { del perfil del } \\
\text { egresado }\end{array}$ & $\begin{array}{c}\text { Áreas } \\
\text { curriculares }\end{array}$ \\
\hline \multicolumn{7}{|c|}{$1 \ldots \ldots$} \\
\hline & $2 \ldots \ldots$ & & & & & \\
\hline
\end{tabular}

Fuente: Santivañez (2013).

TABLA 15. Aspectos enfoque pedagógico y metodológico

\begin{tabular}{|c|c|c|c|c|c|c|}
\hline Código & Asignatura & Condición & Horas teóricas & Horas prácticas & T. horas & Créditos \\
\hline
\end{tabular}

Fuente: Santivañez (2013).

Para la distribución del tiempo formativo se puede emplear el sistema de créditos. El Decreto 4904 de 2009 establece que un crédito equivale a 48 horas, que se distribuyen de dos maneras: horas teóricas y horas prácticas. E1 80 \% de las horas teóricas debe ser presencial; el $20 \%$ se emplea para trabajo extraclase o trabajo autónomo. Por su parte, las horas de prácticas deben ser $100 \%$ presenciales. Es importante resaltar que este decreto establece que como mínimo los programas técnicos laborales deben ser de 600 horas y que el $50 \%$ debe ser de práctica.

Mapa curricular. El mapa muestra de manera lógica la distribución y secuencia de asignaturas a través de un diagrama de flujo o matriz. Aquí se propone como ejemplo la tabla 16.

TABLA 16. Mapa curricular

\begin{tabular}{ll}
\hline Nivel & Asignatura \\
\hline
\end{tabular}

Fuente: Santivañez (2013).

Datos generales de la asignatura. En esta etapa se identifican los datos de la materia: código, departamento, nombre del docente, fecha en el que inicia y finaliza la formación, periodo, duración, prerrequisitos, créditos, extensión horaria y duración.

Formulación de sumillas de asignatura. La sumilla permite enlazar la microprogramación realizada por el docente con el diseño curricular del programa y la institución; esta se realiza con el fin 
de asegurar el cumplimiento de las competencias estipuladas en el perfil de egreso. La redacción debe contener los siguientes elementos: área al que corresponde la asignatura, naturaleza de la asignatura, propósito de la asignatura, unidades de aprendizaje que conforman el contenido de la asignatura y condición de la asignatura (es decir, si es prerrequisito de otra materia).

Formulación del objetivo de la asignatura. En esta etapa se especifica de manera general el qué, el cómo y el para qué de la asignatura. Para establecerlos se debe tener en cuenta los siguientes elementos: conceptual, procedimental y actitudinales. Para la redacción se debe tener en cuenta la taxonomía de objetivos, y seleccionar los verbos dependiendo del dominio de aprendizaje. Para redactar los verbos deber estar en tiempo infinitivo, manteniendo la coherencia y precisión. La tabla 17 explica cada uno de los elementos.

TABLA 17. Formulación del objetivo de la asignatura

\begin{tabular}{cccc}
\hline Verbo & Qué & Cómo & Para qué \\
\hline La acción & El contenido u objeto & $\begin{array}{l}\text { La condición con que se } \\
\text { espera se realice la acción }\end{array}$ & El contenido conceptual \\
\hline
\end{tabular}

Formulación de las competencias especificas de la asignatura. Para la formulación de debe tener en cuenta las competencias definidas en el perfil de egreso, el contenido de unidad y objetivo de la asignatura. En momento de la redacción de debe tener la misma estructura de la formulación del objetivo de asignatura y el verbo debe corresponder al mismo domino y categoría del aprendizaje considerados en el objetivo de la asignatura. Por cada unidad identificada se debe redactar una competencia específica; se recomienda que como mínimo se redacten tres competencias o las necesarias para cumplir con el objetivo de la asignatura.

Organización de la asignatura. En este apartado se incluye un listado de temas y subtemas claves del programa. Para esquematizar los temas se recomienda realizar un mapa conceptual.

Plan de aprendizaje. En esta etapa se especifican las actividades y se determinan las estrategias de evaluación. Este plan se divide de en 4 partes:

- Se formula la competencia del tema y unidad de competencia.

- Se incluyen las estrategias de aprendizaje, para lo cual se especifica el nombre de la actividad, el tipo de mediación, los recursos que se usaran, el procedimiento de trabajo; asimismo, se estipulan las acciones del estudiante y la duración de las actividades del estudiante y de las demás actividades.

- Se formulan las estrategias de evaluación. En esta parte se expresan las evidencias, los instrumentos de evaluación y el valor asignado para cada tema.

- En el último apartado se escribe la bibliografía o fuentes de información.

Normas de convivencia. Con el fin de desarrollar las clases de una manera adecuada se establecen unas normas básicas, como, por ejemplo, puntualidad, asistencia, uso de aparatos electrónicos, fechas de entregable y evaluaciones, entre otros.

Evaluación de los aprendizajes. Los estudiantes deben ser evaluados con el fin de conocer el nivel de desempeño y desarrollo de las competencias definidas en el perfil de egreso. Basándose en los métodos e instrumentos de evaluación definidos en el marco teórico, se propone emplear el uso de rúbricas, ya que estas permiten conocer el desempeño del estudiante durante todo el curso y el desarrollo de actividades por medio de las evidencias de aprendizaje. Para implementar esta estrategia se debe realizar una matriz de valoración, en la que se relacionan un conjunto de criterios 
descritos en una escala de cualificación en función del tipo de competencia que se requiere desarrollar. Para elaborar una rúbrica se deben tener en cuenta las siguientes siete etapas:

1. Establecimiento de la naturaleza del desempeño deseado en términos de las competencias previamente establecidas. En esta etapa se establecen los aspectos a evaluar y las evidencias. Para lo anterior es aconsejable que el docente se responda las preguntas establecidas en a tabla 18 .

TABLA 18. Preguntas naturaleza del desempeño

¿Qué es lo que deseo que mis alumnos sepan? (el qué)

¿Qué es lo que deseo que mis alumnos hagan? (el cómo)

¿Qué es lo que deseo que mis alumnos comprendan? (el para qué)

2. Identificación de las dimensiones o categorias que determinan los aspectos a evaluar de acuerdo a las metas a alcanzar. Se definen los conceptos o tareas que el estudiante debe considerar para el momento de realizar la actividad. Este concepto en conjunto conforma la competencia a desarrollar (se propone emplear la tabla 19 para definirlas).

TABLA 19. Identificación de dimensiones o categorías

\begin{tabular}{lcccc}
\hline & \multicolumn{4}{c}{ Competencia a desarrollar $(\ldots .)}$. \\
\hline Categoría & 1 & 2 & 3 & 4 \\
\hline
\end{tabular}

Fuente: López (2013).

3. Determinación del tipo de escala o niveles de ejecución. Las escalas permiten determinar los niveles requeridos para alcanzar la competencia. Las escalas pueden ser cuantitativas, cualitativas o mixtas.

4. Desarrollo de cada uno de los criterios o descriptores de cada categoría según los niveles de ejecución deseados. Los descriptores permiten definir el nivel de evidencias a alcanzar y reflejan las etapas por las que pasan los estudiantes para desarrollar una competencia. En esta etapa se describe de manera detallada cada uno de los criterios estipulados.

5. Determinación del peso porcentual para cada categoría y criterio. Se asigna un peso en porcentaje a cada categoría, mientras que los criterios cuentan con su propio valor.

6. Desarrollo de las indicaciones. Se redactan las actividades e indicaciones para que el estudiante pueda realizar la actividad.

Requisitos sobre el personal docente, facilitador, tutor, instructor o formador. La institución debe contar con personal que tenga las competencias de formación pedagógica y competencias específicas mínima de un año. Para evaluar el perfil de idóneo de un docente deben tener en cuenta los siguientes criterios:

- Demostrar competencias laborales y especificar del área en que se va desempeñar.

- Demostrar competencias pedagógicas que permitan el desarrollo del plan de estudios.

- Acreditar sus competencias pedagógicas y específicas por medio de una entidad debidamente aprobada por el Ministerio de Educación. 
TABLA 20. Competencias de personal docente

\begin{tabular}{|c|c|c|}
\hline Funciones & Conocimientos & Habilidades \\
\hline $\begin{array}{l}\text { Dirige y facilita el proceso para que los estudiantes elaboren los } \\
\text { productos y servicios propios de la ocupación laboral que están } \\
\text { aprendiendo, con base en el desarrollo de conocimientos, técnicas, } \\
\text { habilidades, destrezas y actitudes. } \\
\text { - Planificar sesiones de aprendizaje, demostraciones prácticas, talleres, } \\
\text { seminarios y trabajo en equipo. } \\
\text { - Evaluar el trabajo del estudiante, preparando, aplicando y calificando } \\
\text { pruebas de verificación del aprendizaje. } \\
\text { Diseñar programas curriculares y preparar material didáctico para el } \\
\text { aprendizaje. } \\
\text { - Suministrar orientación a los estudiantes sobre alternativas de carrera } \\
\text { u oficio } \\
\text { requisitos de ingreso y certificación. }\end{array}$ & $\begin{array}{l}\text { - Educación y } \\
\text { capacitación } \\
\text { - Idioma extranjero } \\
\text { - Servicio al cliente } \\
\text { - Administración y } \\
\text { gerencia } \\
\text { - Sicología }\end{array}$ & $\begin{array}{l}\text { - Estrategias para } \\
\text { el aprendizaje } \\
\text { - Transmisión de } \\
\text { conocimiento } \\
\text { - Comunicación } \\
\text { asertiva } \\
\text { - Escucha activa } \\
\text { - Comprensión de } \\
\text { lectura }\end{array}$ \\
\hline
\end{tabular}

Fuente: Observatorio Laboral y Ocupacional Colombiano (2015).

Distribución de la asignación académica para el personal docente. La institución debe contar con criterios que permitan realizar la asignación académica con el fin de establecer el número de docentes por estudiantes. Los criterios que se debe tener en cuenta son los siguientes:

- Estándares según normatividad

- Número de estudiantes inscritos

- Complejidad de las competencias a desarrollar

Según el Decreto 1075, el número mínimo de estudiantes en una zona urbana es de 32 (esto depende del centro de educación). Para educación media técnica, la normativa establece por grupo debe haber 1.7 docentes. Según la complejidad de las competencias a desarrollar en la institución, se propone tener un máximo de 15 estudiantes por docente, ya que el nivel de acompañamiento por parte del docente debe ser permanente y activo; esta actividad se debe monitorear contantemente y tiene que basarse en indicadores y encuestas de satisfacción.

Vinculación en sector productivo. Con el fin de vincular a los egresados de la institución, mantener un continuo monitoreo del desempeño y consultar el perfil de egreso del estudiante, se debe realizar acuerdos con el sector productivo al que apuntan, de tal manera que fomenten el mejoramiento de los aspectos que afectan el resultado de las competencias a desarrollar. Para lo anterior se propone que la institución realice convenios de cooperación en los que se establezcan los siguientes puntos:

- Prácticas de los estudiantes en entornos reales.

- Capacitación de lo personal docente.

- Evaluación del desempeño de los egresados y practicantes.

- Acciones de formación en espacios productivos.

Es importante que la institución mantenga registros donde se evidencien los vínculos establecidos. 
Recursos especificos del programa. Para desarrollar cada las competencias estipuladas en el perfil de egreso, la institución debe contar con recursos necesarios, de acuerdo al Decreto 1075 en el titulo 4, numeral 9.

\section{Etapa de hacer}

En esta etapa se explica cómo realizar el proceso de capacitación e implementación, con el fin de lograr que lo planeado sea puesto de práctica en el entorno educativo. Para lograrlo se propone un plan de capacitación dividido en módulos y etapas que tienen unos temas actividades asignadas, las cuales permiten que se realicen acciones de implementación a medida que se avanza en la capacitación.

En el primer módulo se explica la importancia de la capacitación y el proceso de implementación. Posteriormente se explican los objetivos, contenidos y las actividades de la capacitación, para lo que se proponen los siguientes objetivos:

- Realizar la justificación de los programas basada en el análisis del contexto.

- Establecer el perfil de egreso en base en la metodología el formato propuesto.

- Diseñar el plan de estudios apoyado en la metodología propuesta y el enfoque pedagógico por competencias.

Para cumplir con el anterior, se establece un programa de capacitación e implementación donde asignarán actividades que permitirán ir desarrollando la propuesta planteada.

TABLA 21. Cronograma de capacitación e implementación

\begin{tabular}{|c|c|c|c|c|c|}
\hline \multirow[b]{2}{*}{ Módulo } & \multirow[b]{2}{*}{ Tema } & \multirow[b]{2}{*}{ Subtema } & \multirow[b]{2}{*}{ Actividad } & \multicolumn{2}{|c|}{ Duración } \\
\hline & & & & Sesiones & $\begin{array}{l}\text { Horas } \\
\text { totales }\end{array}$ \\
\hline 1 & $\begin{array}{l}\text { Presentación y } \\
\text { justificación }\end{array}$ & $\begin{array}{l}\text { - Análisis del entorno } \\
\text { - Revisión del marco legal y } \\
\text { normativo } \\
\text { - Análisis de la oferta y tendencias } \\
\text { educativas. } \\
\text { - Análisis DOFA }\end{array}$ & $\begin{array}{l}\text { - Matriz DOFA } \\
\text { - Realizar la } \\
\text { justificación del } \\
\text { programa }\end{array}$ & 3 sesiones & 6 \\
\hline 2 & $\begin{array}{l}\text { Definir perfil de } \\
\text { egreso e ingreso }\end{array}$ & $\begin{array}{l}\text { Definición de competencias. } \\
\text { - Explicación de la metodología } \\
\text { definición de perfil }\end{array}$ & $\begin{array}{l}\text { Matriz de perfil de } \\
\text { ingreso y egreso }\end{array}$ & 3 sesiones & 6 \\
\hline 3 & $\begin{array}{l}\text { Desarrollar el } \\
\text { plan de estudios }\end{array}$ & $\begin{array}{l}\text { - Aprendizaje basado en } \\
\text { competencias. } \\
\text { - Explicación de la metodología del } \\
\text { plan de estudios. } \\
\text { - Explicación de actividades de } \\
\text { aprendizaje } \\
\text { - Explicación de método de } \\
\text { evaluación. }\end{array}$ & $\begin{array}{l}\text { - } \text { Creación de } \\
\text { asignaturas } \\
\text { - Mapa de } \\
\text { asignaturas } \\
\text { - Formulación de la } \\
\text { - } \text { asignatura } \\
\text { - } \text { actividades } \\
\text { - Métodos de } \\
\text { evaluación } \\
\text { - Plan de estudios }\end{array}$ & 6 sesiones & 12 \\
\hline 4 & $\begin{array}{l}\text { - Distribución } \\
\text { asignación } \\
\text { académica }\end{array}$ & $\begin{array}{l}\text { Explicación de la normatividad y } \\
\text { marco legal }\end{array}$ & $\begin{array}{l}\text { - Cuadro de } \\
\text { distribución de } \\
\text { asignación }\end{array}$ & 1 sesión & 2 \\
\hline
\end{tabular}




\begin{tabular}{|c|c|c|c|c|c|}
\hline \multirow[b]{2}{*}{ Módulo } & \multirow[b]{2}{*}{ Tema } & \multirow[b]{2}{*}{ Subtema } & \multirow[b]{2}{*}{ Actividad } & \multicolumn{2}{|c|}{ Duración } \\
\hline & & & & Sesiones & $\begin{array}{c}\text { Horas } \\
\text { totales }\end{array}$ \\
\hline \multirow[t]{2}{*}{5} & $\begin{array}{l}\text {-Definición } \\
\text { perfil personal } \\
\text { docente. }\end{array}$ & $\begin{array}{l}\text { - Explicación de la normatividad y } \\
\text { marco legal }\end{array}$ & $\begin{array}{l}\text { - Definición del } \\
\text { perfil y método } \\
\text { de entrevista del } \\
\text { personal docente. }\end{array}$ & 1 sesión & 2 \\
\hline & & & TOTAL & 14 & 28 \\
\hline
\end{tabular}

\section{Etapa de verificación}

La etapa de verificación permite realizar seguimiento a las actividades propuestas en la etapa de planificación, lo que permite evaluar los resultados de diseño curricular, el uso de recursos y la satisfacción de las partes interesadas. En esta etapa se definen los indicadores, los cuales permiten medir el desempeño del logro de los objetivos.

Considerando que la institución no tiene un sistema de gestión de calidad y no ha definido sus actividades por procesos, se propone la tabla 22 para definir proceso y objetivo y diseñar los indicadores.

TABLA 22. Objetivo del proceso de formación

\section{Objetivo del proceso de formación}

Desarrollar competencias en los estudiantes según el perfil de egreso, apoyadas en actividades, recursos y personal docente pertinente, de una manera oportuna y eficaz.

Una vez establecido en objetivo del proceso, se propone establecer métodos de seguimiento y recopilación de información, acorde con:

- Satisfacción del cliente

- Desempeño del proceso

- Desempeño del personal docente

- Resultados de los egresados

- Desempeño del programa

Para lo anterior se propone los métodos señalados en la tabla 23. En este cuadro se establece el nombre del indicador, fuente de obtención de datos, unidad de medida, frecuencia, formula y meta. Por otro lado, para obtener información sobre la satisfacción del cliente, desempeño del docente y desempeño del programa se propone emplear: encuestas de satisfacción del programa, evaluación de docentes y encuestas del desempeño de los egresados en el sector productivo. Una vez realizadas las encuestas se puede obtener información, evaluar los resultados e implementar acciones de mejoras. 
TABLA 23. Indicadores de gestión proceso de formación

\begin{tabular}{|c|c|c|c|c|}
\hline $\begin{array}{l}\text { Nombre del } \\
\text { indicador }\end{array}$ & $\begin{array}{l}\text { Fuente obtención } \\
\text { de datos }\end{array}$ & $\begin{array}{l}\text { Unidad de } \\
\text { medida }\end{array}$ & Frecuencia & Fórmula de cálculo. \\
\hline $\begin{array}{l}\text { Cumplimento de } \\
\text { cronograma de } \\
\text { actividades }\end{array}$ & $\begin{array}{l}\text { Cronograma de } \\
\text { actividades. } \\
\text { Fechas inicio y } \\
\text { finalización de la } \\
\text { actividad }\end{array}$ & $\%$ & $\begin{array}{l}\text { Cada vez } \\
\text { que se } \\
\text { finaliza el } \\
\text { modulo }\end{array}$ & {$\left[\frac{\text { Tiempo de empleado }}{\text { Tiempo programado }}\right] \times 100 \%$} \\
\hline $\begin{array}{l}\text { Índice de } \\
\text { deserción de } \\
\text { estudiantes }\end{array}$ & $\begin{array}{l}\text { Bases de datos } \\
\text { matriculas }\end{array}$ & $\%$ & Mensual & {$\left[\frac{\text { No. de est. actuales en cada programa }}{\text { No. de est. matriculados cada programa }}\right] \times 100-100$} \\
\hline $\begin{array}{l}\text { Índice de } \\
\text { asistencia }\end{array}$ & $\begin{array}{l}\text { Planillas de } \\
\text { asistencia }\end{array}$ & $\%$ & Mensual & {$\left[\frac{\text { No. est. } \times \text { dias clase }}{\text { No. estudiantes } \times \text { dias de clase }}\right] \times 100 \%$} \\
\hline $\begin{array}{l}\text { Promoción } \\
\text { estudiantil }\end{array}$ & $\begin{array}{l}\text { Registro de } \\
\text { grados }\end{array}$ & $\%$ & $\begin{array}{l}\text { Cada } \\
\text { periodo }\end{array}$ & {$\left[\frac{\text { No. de est. graduados en cada programa }}{\text { No. de est. matriculados cada semestre }}\right] \times 100 \%$} \\
\hline $\begin{array}{l}\text { Índice de } \\
\text { vinculación } \\
\text { laboral }\end{array}$ & $\begin{array}{l}\text { Bases de datos } \\
\text { egresados }\end{array}$ & $\%$ & $\begin{array}{l}\text { Cada } \\
\text { periodo }\end{array}$ & {$\left[\frac{\text { No. de est. vinculados al } \text { sector productivo }}{\text { No. de est. egresados }}\right] \times 100 \%$} \\
\hline
\end{tabular}

\section{Etapa actuar}

En esta etapa se definen las acciones de mejora basadas en los datos recopilados en la sección de verificación, los cuales permiten definir y evaluar la toma de decisiones con respecto al proceso de mejora. Para lo anterior se propone una serie de pasos que permitan de una manera secuencial y continua mejorar los programas de formación.

- Paso 1. Definir e identificar oportunidades de mejora. Para realizar esta etapa se debe considerar las fuentes de datos recopiladas en la etapa de verificación, es decir, las encuestas e indicadores de gestión.

- Paso 2. Describir o redactar la acción de mejora. Este paso se realiza con base en el análisis de la información recopilada. Se debe redactar las oportunidades demostrando la intención, el estado actual y el estado deseado.

- Paso 3. Establecer acciones de mejora. Las acciones de mejora deben ser apropiadas a los objetivos propuestos, aumentar la eficiencia y eficacia de los programas de formación y producir rentabilidad en la institución. Para este paso se propone la tabla 24.

TABLA 24. Formato de acciones de mejora

\begin{tabular}{ccccccc}
\hline $\begin{array}{c}\text { Oportunidad de } \\
\text { mejora }\end{array}$ & $\begin{array}{c}\text { Acción de } \\
\text { mejora }\end{array}$ & $\begin{array}{c}\text { Recursos } \\
\text { requeridos }\end{array}$ & Responsable & \multicolumn{2}{c}{ Seguimiento } & Criterios de \\
& mecha 1 & Fecha 2 & mejora \\
\hline
\end{tabular}

- Paso 4. Revisar las acciones tomadas. Una vez establecidas e implementadas las acciones de mejora, es importan determinar si estas fueron apropiadas, para lo cual se deben evaluar con respecto al criterio definido y a la nueva recopilación de datos obtenidos al iniciar el ciclo PHVA. 


\section{Conclusiones}

El desarrollo y diseño de un programa de formación es una actividad muy importante dentro del acto educativo, ya que en la fase de planeación se tienen en cuenta las competencias necesarias para desempeño ideal de la profesión, es decir, de define el perfil de egreso. Adicional a lo anterior y una vez definido el perfil, se crean las actividades y el método de evaluación que permitan desarrollar las habilidades y compresión de los estudiantes con respecto de los temas tratados; lo que busca es vincular a los estudiantes al mundo productivo y mejorar la calidad de vida del egresado.

$\mathrm{El}$ aprendizaje basado en competencias es un enfoque que busca desarrollar al estudiante en tres dimensiones: el saber ser, el saber comprender y el saber hacer, con lo que se pretende que el alumno comprenda y resuelva problemas del sector, se comporte de manera adecuada según el contexto y sepa desenvolverse de manera correcta según las actividades asignadas. Este enfoque permite formar seres humanos íntegros, responsables y proactivos que aporten al desarrollo del país, de su comunidad y de su agremiación.

La metodología propuesta orienta sus elementos en el estudiante y requiere que el docente diseñe experiencias que permitan desarrollar competencias y habilidades y consideren la forma cómo aprenden los seres humanos; esto quiere decir que es un enfoque que requiere mayor grado participación de los estudiantes y que no se limita a la exposición de conceptos y ni a la memorización de temas.

La certificación de programas de formación busca que la institución demuestre y mejore la capacidad para cumplir con las necesidades de formación, ya que la metodología planteada permite que en el momento de realizar el diseño del programa se parta de las competencias que requiere el sector productivo para desempeñarse de manera adecuada. También se deben tener cuenta las competencias básicas y ciudadanas, la cuales permiten convivir en sociedad y mantener el aprendizaje a lo largo de la vida.

La implementación y certificación de los programas en instituciones de formación para el trabajo y el desarrollo humano es un proceso dispendioso e implica altos costos para este tipo de organizaciones, ya que en su mayoría son microempresas, por tal motivo se requieren esfuerzos financieros significativos (este puede ser uno de los factores que explica el poco porcentaje de instituciones certificadas). Por otro lado, si se piensa a largo plazo y el sistema se implementa de manera coherente con los elementos estratégicos de la organización, los ingresos, las utilidades y el crecimiento pueden ser mayores al del promedio del sector, lo que se puede convertir en una oportunidad que contribuya a que las instituciones sean más competitivas.

\section{REFERENCIAS}

Decreto 4904 de 2009. Por el cual se reglamenta la organización, oferta y funcionamiento de la prestación del servicio educativo para el trabajo y el desarrollo humano y se dictan otras disposiciones. Diario Oficial n. ${ }^{\circ} 47567$ del 18 de diciembre de 2009.

Decreto 1075 de 2015. Por medio del cual se expide del decreto único reglamentario del sector educación. Diario Oficial n. ${ }^{\circ} 4923$ del 26 de mayo de 2015.

Díaz, J. (2013). Calidad educativa: Un análisis sobre la acomodación de los sistemas de gestión de la calidad empresarial a la valoración en educación. Tendencias Pedagógicas, 21, 177-194.

Icontec. (2011a). Norma técnica colombiana 5555. Sistema de gestión de calidad para instituciones de formación para el trabajo. Bogotá D. C.: Icontec. 
Icontec. (2011b). Norma técnica colombiana 5581. Programas de formación para el trabajo. Requisitos. Bogotá D. C.: Icontec.

Icontec. (2015a). Norma técnica colombiana 9000. Sistemas de gestión de la calidad, fundamentos y vocabulario. Bogotá D. C.: Icontec.

Icontec. (2015b). Norma técnica colombiana 9001. Sistemas de gestión de la calidad. Requisitos. Bogotá D.C.: Icontec.

López, M. (2013). Aprendizaje, competencias y TIC (1ª edición). México: Pearson.

Ministerio de Educación Nacional. (2016). Educación superior- evaluación, certificación y acreditación. Recuperado de http://www.mineducacion.gov.co/1759/w3 -article-307592.html

Observatorio Laboral y Ocupacional Colombiano. (2015). Clasificación nacional de ocupaciones C.N.O. Bogotá: Sena, Mintrabajo.

Ortiz, Y., y Ramírez M. (2017). Avances de las certificaciones ISO 9001 e ISO 14001 en Colombia. Revista chilena de economía y sociedad, 39-53.

Pimienta, J. (2012). Las competencias en la docencia universitaria (1ª edición). México: Pearson.

Proyecto Tuning América Latina. Recuperado de http://tuning.unideusto.org/tuningal/

Soin, S. (1997). Control de calidad total: claves, metodologías y administración para el éxito. México: McGraw-Hill Interamericana.

Santivañez, V. (2013). Diseño curricular a partir de competencias (2ª edición). Bogotá: Ediciones la U. 